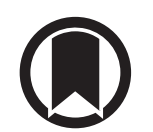

CrossMark

\title{
NETs: a new biomarker of traffic-related air pollution exposure: are they ready to catch fish?
}

\author{
Howard M Kipen ${ }^{1,2}$ and Debra L. Laskin ${ }^{2,3}$ \\ Affiliations: ${ }^{1}$ Dept of Environmental and Occupational Health, Rutgers University, School of Public Health, \\ Piscataway, NJ, USA. ${ }^{2}$ Environmental and Occupational Health Sciences Institute (EOHSI), Piscataway, NJ, \\ USA. ${ }^{3}$ Dept of Pharmacology and Toxicology, Rutgers University, Ernest Mario School of Pharmacy, \\ Piscataway, NJ, USA.
}

Correspondence: Howard M. Kipen, Dept of Environmental and Occupational Health, Rutgers University, School of Public Health, Environmental and Occupational Health Sciences Institute (EOHSI), 170 Frelinghuysen Road, Piscataway, NJ, 08854, USA. E-mail: kipendeohsi.rutgers.edu

@ERSpublications

There are many challenges in interpretation of novel assays for health effects in human challenge studies https://bit.ly/3bdF5me

Cite this article as: Kipen HM, Laskin DL. NETs: a new biomarker of traffic-related air pollution exposure: are they ready to catch fish? Eur Respir J 2020; 55: 2000305 [https://doi.org/10.1183/13993003.00305-2020].

Air pollution has been with humankind throughout evolution (e.g. volcanoes, forest fires), but it rapidly accelerated with the industrial revolution. Although the history of London's suffocating fogs began in the 18th century with coal power, it was the acute London fog episode of December 1952, and similar crises, that galvanised public health officials to begin to address the problem. The tremendous growth of automobiles reliant on internal combustion engines during the latter half of the 20th century ultimately led to current concerns with traffic-related air pollution (TRAP), causing researchers to seek a better understanding of air pollution health effects $[1,2]$. The importance of this for public heath became key when the Harvard Six Cities Study and others demonstrated that particulate matter air pollution, not visible to the naked eye and producing minimal acute signs or symptoms, was strongly associated with mortality and serious morbidity [3-5]. To explain and support these epidemiological findings, more sophisticated human toxicology studies became essential for the mechanistic research that could provide stronger evidence of a causal association [6]. In this regard, early human controlled exposure studies with pollutant gases, primarily ozone and sulfur dioxide, provided some clues on how air pollutants caused morbidity and mortality [7-9].

Controlled human studies have generally involved acute exposures with relatively short follow-up (usually less than $24 \mathrm{~h}$ ) and relied on biomarker endpoints. Presently, there are at least four human inhalation facilities in North America and one in Europe that utilise fresh diesel exhaust (DE) to generate particles for mechanistic experiments, with maximum concentrations benchmarked to those reported at the highest ambient levels (e.g. bus stations). Endpoints that have been investigated in controlled studies with DE include cellular, biochemical, oxidative stress, and other markers of pulmonary and systemic inflammation [10]. A group at Umea University in Sweden pioneered fresh DE exposures and the use many of these endpoints, as well as more invasive arterial plethysmographic and bronchoscopic sampling techniques [11]. Studies in clinically susceptible populations such as asthmatics or survivors of myocardial infarction have also been safely performed and results contribute to understanding of pathophysiologic mechanisms and regulatory guidelines. Pertinent to the current study is a 2001 paper that reported increased 
interleukin-8 and polymorphonuclear leukocytes (PMNs) in peripheral blood of healthy volunteers, but not in (vulnerable) asthmatics following DE exposure [12].

It is against this experimental background that the present study reports on a potential new inflammatory biomarker, neutrophil extracellular traps (NETs). In studies by Wooding et al. [13], never-smokers $(n=7)$, ex-smokers $(n=4)$ and mild-moderate COPD patients $(n=7)$ were exposed to DE $\left(300 \mu \mathrm{g} \cdot \mathrm{m}^{-3}\right)$ or filtered air for $2 \mathrm{~h}$ with intermittent exercise on separate occasions at least 4 weeks apart in a crossover study design. DE was found to reduce peripheral blood band cells, but to increase neutrophil activation, responses exaggerated in COPD patients, relative to never-smokers. These findings are important as they indicate increased susceptibility to particulate matter air pollution among those with pre-existing respiratory conditions [14]. DE was also found to increase the release of NETs in vitro by peripheral blood neutrophils from healthy and asthmatic subjects, with no stated differences between the two subject groups. Similarly, although numbers of NETs present in bronchoalveolar lavage fluid increased after DE exposure, there were no differences between never smokers, ex-smokers and COPD patients. The authors conclude that NET release is a possible mechanism to explain how TRAP contributes to airway pathophysiology, but not to the increased susceptibility of vulnerable individuals.

Neutrophil extracellular traps (NETs) are structures consisting of DNA, histones and intracellular enzymes which are released from neutrophils during a process referred to as NETosis. Originally thought to be involved primarily in the capture of pathogens, more recent evidence suggests that NETs are also released during sterile inflammatory responses; in this context, NETs have been implicated in a variety of non-infectious lung diseases including asthma, COPD, cystic fibrosis, and cancer, and in acute injury induced by cigarette smoke, e-cigarettes, titanium dioxide, nanoparticles, lipopolysaccharide, uric acid, and mechanical ventilation [15-25]. However, whether NETs play a beneficial or detrimental role in host defence against non-infectious, as well as infectious stimuli, depends on carefully controlled release and removal of NETs [26]. In the absence of this control, histones, antimicrobial peptides and proteolytic enzymes decorating NET-DNA, which may possess nonspecific cytotoxic activity, can cause deleterious inflammation and collateral tissue injury [17]. It has been suggested that the detrimental effects of excessive NET release are particularly important in lung pathology, because NETs can expand more easily in the pulmonary alveoli, causing greater tissue injury [18]. In this regard, extracellular histones within NETs have been reported to directly cause lung epithelial and endothelial cell death [27, 28].

Two major NETosis pathways have been described (figure 1): suicidal NETosis, which involves plasma membrane rupture and cell death; and vital NETosis, in which the cells, although anuclear, remain viable and are capable of chemotaxis and phagocytosis [28-31]. A third pathway of NETosis involves the release of mitochondrial DNA; however, this is thought to occur mainly in eosinophils. While NET release without cell death (vital NETosis) occurs rapidly following exposure to inflammatory stimuli (within $\sim 5-$ $30 \mathrm{~min}$ in cultured cells), suicidal NETosis occurs over a period of hours and is dependent on NADPH oxidase and production of reactive oxygen species. Whether these different types of NETosis occur in different subsets of neutrophils or are activated by distinct stimuli remains to be determined. It is worth noting, however, that only $20-25 \%$ of neutrophils release NETs following stimulation $[17,18]$.

The concept that exposure to particulate air pollution (i.e. DE) causes human neutrophil activation and the release of NETs is important as it suggests a potential pathogenic mechanism by which inflammatory neutrophils can contribute to tissue injury and disease pathogenesis. However, some caution is needed in interpreting results and drawing conclusions from this study, as there are technical issues related to the methods used to assay extracellular NETs. The flow cytometric method used for analysis of NETs is based on a method published by GAVILLET et al. [32]. As previously noted in a letter to the editor regarding the Gavillet paper, there are methodological limitations to this assay [33]. These include clumping of cells due to the presence of highly viscous DNA, making it difficult to assess individual cells in suspension by flow cytometry. Additionally, as NETs are, by definition, extracellular structures, it is somewhat unclear how to interpret results based on gating intact cells. In this regard, distinguishing between NETs that are released from neutrophils during suicidal NETosis, typically associated with "sterile" inflammation, and viable neutrophils as they rapidly respond to bacterial pathogens [28-31], would be valuable.

Immunocytochemistry was performed to assess NETosis by bronchoalveolar lavage cells from human subjects. DE exposure reportedly increased the amount of NETs released; however, there were no effects of patient phenotype or order of exposure. While these findings are potentially important, more detailed description of the methods and figures would facilitate data interpretation. Information about the percentage of macrophages in bronchoalveolar lavage would also be valuable. Macrophages are known to release extracellular DNA decorated with histones and proteases, in a process referred to as METosis [34]; they also harbour neutrophil elastase both intracellularly and on their surfaces. 
Suicidal NETosis

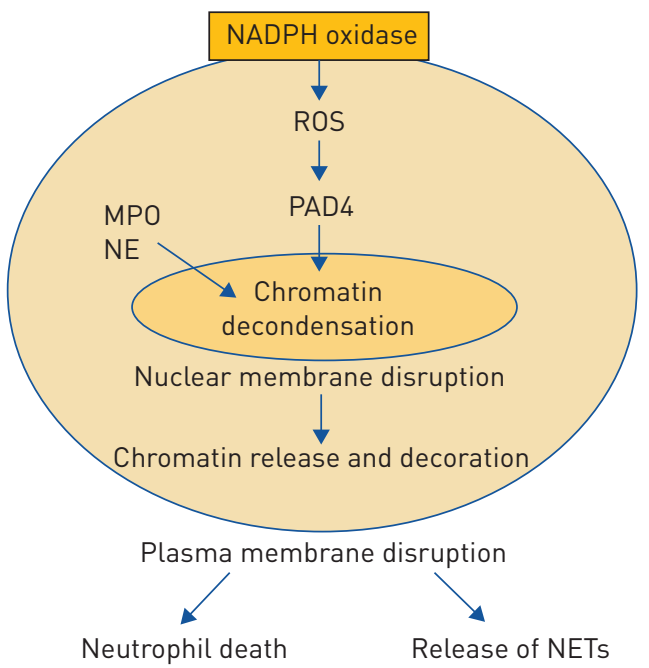

Vital NETosis

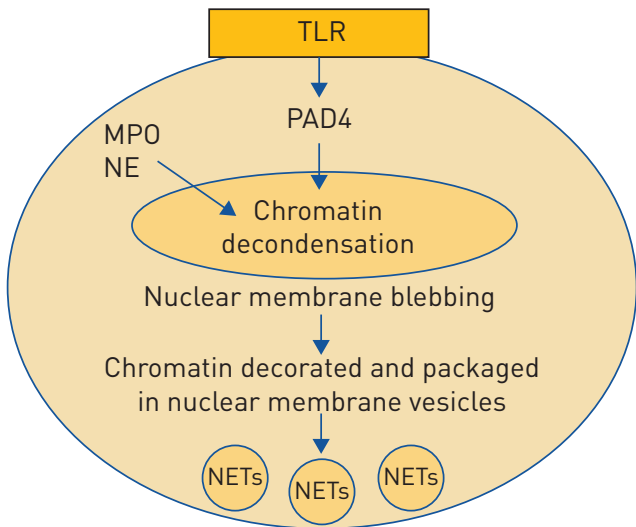

No plasma membrane disruption

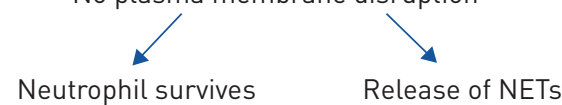

FIGURE 1 Polymorphonuclear leukocyte (PMN) neutrophil NETosis. Left: Suicidal NETosis. A key initiating step in suicidal NETosis is activation of NADPH oxidase and reactive oxygen species (ROS) production. ROS cause activation of peptidyl arginine deiminase 4 (PAD4) and rupture of cytoplasmic granules, releasing myeloperoxidase (MPO) and neutrophil elastase (NE). MPO and NE translocate to the nucleus and together with PAD4 cause chromatin decondensation. This leads to rupture of the nuclear membrane and release of chromatin into the cytoplasm where it is decorated with granular and cytoplasmic proteins. Neutrophil extracellular traps (NETs) are released following plasma membrane rupture. This process typically requires several hours and culminates in PMN lysis and death. Right: Vital NETosis. Following activation of Toll-like receptors (TLRs) by pathogens and/or platelets, PAD4 is activated and NE and MPO released causing chromatin decondensation. Chromatin is decorated and released into the cytoplasm within vesicles formed by blebbing of the nuclear membrane, a process which ultimately leads to nuclear membrane rupture. Vesicles containing NETs are released from PMN via exocytosis leaving the plasma membrane intact and the anuclear PMN capable of phagocytosis and chemotaxis. This process occurs within minutes of initiation.

Recruitment of subjects across such broad age ranges (40-80 years), as well as underrepresentation of women also limits the external validity of this report. Notwithstanding the substantial challenges of subject recruitment in delimited disease phenotypes, these demographics are important to consider. It is well established that ageing is associated with low-grade chronic sterile inflammation (inflammaging) [35]; this baseline activation state of inflammatory cells in the elderly can significantly alter the response to external stimuli. In this context, the majority of elderly subjects, logically, fell within the COPD group, which was also more sensitive to DE. Gender differences in immune and inflammatory responses, and in response to air pollutants, are also well established [36]. Studies with increased numbers of subjects and a consideration of demographic susceptibilities will broaden the impact of this novel and potentially newest mechanistic air pollution biomarker of effect.

Support statement: Supported by National Institutes of Health grants: ES005022, ES004738, AR055073 and TR0030117, and US EPA R832144.

Conflict of interest: H.M. Kipen has nothing to disclose. D.L. Laskin has nothing to disclose.

\section{References}

Corton C. London Fog: The Biography. Cambridge, Belknap Press, 2017.

Kovarik B. Environmental History Timeline: Air Pollution. http://environmentalhistory.org/about/airpollution Dockery DW, Pope CA 3rd, Xu X, et al. An association between air pollution and mortality in six U.S. cities. N Engl J Med 1993; 329: 1753-1759.

4 Pope CA, Thun MJ, Namboodiri MM, et al. Particulate air pollution as a predictor of mortality in a prospective study of U.S. adults. Am J Respir Crit Care Med 1995; 151: 669-674.

Dockery DW. Health effects of particulate air pollution. Annals Epidemiol 2009; 4: 257-263.

Hill AB. The environment and disease: association or causation? 1965. Proc Royal Soc Med 1965; 58: 295-300.

Bates DV, Bell G, Burnham C, et al. Problems in studies of human exposure to air pollutants. Can Med Assoc J 1970; 103: 833-837.

8 McDonnell WF, Horstman DH, Hazucha MJ, et al. Pulmonary effects of ozone exposure during exercise: dose-response characteristics. J Appl Physiol Respir Environ Exercise Physiol 1983; 54: 1345-1352.

9 Hazucha MJ, Ginsberg JF, McDonnell WF, et al. Effects of $0.1 \mathrm{ppm}$ nitrogen dioxide on airways of normal and asthmatic subjects. J Appl Physiol Respir Environ Exercise Physiol 1983; 54: 730-739. 
10 Thurston GD, Kipen HM, Annesi-Maesano I, et al. A joint ERS/ATS policy statement: what constitutes an adverse health effect of air pollution? An analytical framework. Eur Respir J 2017; 49: 1600419.

11 Rudell B, Sandstrom T, Hammarstrom U, et al. Evaluation of an exposure setup for studying the effects of diesel exhaust in humans. Int Arch Occup Environ Health 1994; 66: 77-83.

12 Salvi S, Blomberg A, Rudell B, et al. Acute inflammatory responses in the airways and peripheral blood after short-term exposure to diesel exhaust in healthy human volunteers. Am J Respir Crit Care Med 2001; 164: 831-833.

13 Wooding DJ, Ryu MH, Li H, et al. Acute air pollution exposure alters neutrophils in never-smokers and at-risk humans. Eur Respir J 2020; 55: 1901945

14 Zanobetti A, Bind MA, Schwartz J. Particulate air pollution and survival in a COPD cohort. Environ Health 2008; 7: 48 .

15 Bokaba RP, Anderson R, Theron AJ, et al. Cigarette smoke condensate attenuates phorbol ester-mediated neutrophil extracellular trap formation. Afr Health Sci 2017; 17: 896-904.

16 Chézeau L, Kohlstaedt LA, Le Faou A, et al. Proteomic analysis of bronchoalveolar lavage fluid in rat exposed to $\mathrm{TiO}_{2}$ nanostructured aerosol by inhalation. J Proteomics 2019; 207: 103451.

17 Cheng O, Palaniyar N. NET balancing: a problem in inflammatory lung diseases. Front Immunol 2013; 4: 1-13.

18 Porto B, Stein R. Neutrophil extracellular traps in pulmonary disease. Too much of a good thing? Frontiers Immunol 2016; 7: 1-13.

19 Liu T, Wang F-P, Wang G, et al. Role of neutrophil extracellular traps in asthma and chronic obstructive pulmonary disease. Chinese Med J 2017; 130: 730-736.

20 Liu S, Su X, Pan P, et al. Neutrophil extracellular traps are indirectly triggered by lipopolysaccharide and contribute to acute lung injury. Sci Rep 2016; 6: 37252

21 Li H, Pan P, Su X, et al. Neutrophil extracellular traps are pathogenic in ventilator-induced lung injury and partially dependent on TLR4. Biomed Res Int 2017; 2017: 8272504

22 Reidel B, Radicioni G, Clapp PW, et al. E-Cigarette use causes a unique innate immune response in the lung, involving increased neutrophilic activation and altered mucin secretion. Am J Respir Crit Care Med 2018; 197: 492-501.

23 Sabbione F, Keitelman IA, Iula L, et al. Neutrophil extracellular traps stimulate proinflammatory responses in human airway epithelial cells. J Innate Immunol 2017; 9: 387-402.

24 Muñoz LE, Bilyy R, Biermann MH, et al. Nanoparticles size-dependently initiate self-limiting NETosis-driven inflammation. Proc Natl Acad Sci USA 2016; 113: E585.

25 Zou Y, Chen X, He B, et al. Neutrophil extracellular traps induced by cigarette smoke contribute to airway inflammation in mice. Exp Cell Res 2020; 3: 111888.

26 Boeltzl S, Amini P, Anders H-J. To NET or not to NET: current opinions and state of the science regarding the formation of neutrophil extracellular traps. Cell Death Different 2019; 26: 395-408.

$27 \mathrm{Xu}$ J, Zhang X, Pelayo R, et al. Extracellular histones are major mediators of death in sepsis. Nat Med 2009; 15: 1318-1321.

28 Saffarzadeh M, Juenemann C, Queisser M, et al. Neutrophil extracellular traps directly induce epithelial and endothelial cell death: a predominant role of histones. Plos One 2012; 7: e32366.

29 Yipp B, Kubes P. NETosis: how vital is it? Blood 2013; 122: 2784-2794.

30 Delgado-Rizo V, Martinez-Guzman M, Iniguez-Gutierrez L. Neutrophil extracellular traps and its implications in inflammation: an overview. Front Immunol 2017; 8: 81.

31 Yang H, Biermann M, Braumer J, et al. New insights into neutrophil extracellular traps. Mechanisms of formation and role in inflammation. Front Immunol 2016; 7: 302.

32 Gavillet M, Martinod K, Renella R, et al. Flow cytometric assay for direct quantification of neutrophil extracellular traps in blood samples. Amer J Hematol 2015; 90: 1155-1158.

33 Handzlik A, Ostafin M, Bystrzcka W, et al. Flow cytometric quantification of neutrophil extracellular traps: Limitations of the methodological approach. Am J Hematol 2016; 91: E9-E10.

34 Daniel C, Leppkes M, Muñoz LE, et al. Extracellular DNA traps in inflammation, injury and healing. Nat Rev Nephrol 2019; 15: 559-575.

35 Franceschi C, Garagnani P, Parini P, et al. Inflammaging: a new immune-metabolic viewpoint for age-related diseases. Nat Rev Endocrinol 2018; 14: 576-590.

36 Clougherty JE. A growing role for gender analysis in air pollution epidemiology. Environ Health Perspect 2010; 118: $167-176$. 